\title{
SEARCHES FOR EFFECTS OF FLAVOR-CHANGING NEUTRAL CURRENTS hै
}

\author{
G.L. KANE and R. THUN \\ Randall Laboratory of Physics, University of Michigan, Ann Arbor, MI 48109, USA
}

Received 7 April 1980

Revised manuscript received 8 May 1980

\begin{abstract}
We discuss some possible processes, mainly decays, to search for flavor-changing neutral currents. We examine the theoretical constraints from present data as well as improved sensitivities which might be achieved in future experiments. We focus on the decays $\mathrm{K}^{0} \rightarrow \mu \mathrm{e}, \Sigma^{+} \rightarrow$ p $\mu \mathrm{e}, \tau \rightarrow \mu \mu \mathrm{e}$ and $\mu \rightarrow$ eee as being particularly interesting for further experimental searches.
\end{abstract}

1. Introduction. At present the most interesting issues in particle physics include grand unification of the various interactions, mechanisms for symmetry breaking, and accounting for the number of particle "flavors". The existence of flavor, that is the replication of both leptons and quarks, is perhaps the least understood of these issues [1] and is the topic of this paper. Because of our lack of theoretical understanding, we concentrate our efforts on an evaluation of possible experiments to search for rate flavor-changing processes. We base theoretical comparisons of sensitivity on the assumption that such processes are mediated by heavy gauge bosons. The $\mathrm{K}_{\mathrm{L}}-\mathrm{K}_{\mathrm{S}}$ mass difference and the present branching ratio limit of $\mathrm{K}_{\mathrm{L}} \rightarrow \mu \mathrm{e}$ are used to place limits on $g^{2} / m^{2}$, where $g$ is the coupling strength and $m$ the mass of the heavy bosons. These limits from the kaon system are then applied to other decay processes and discussed in relation to possible experimental sensitivities.

We emphasize that our quantitative estimates as well as our conclusions are subject to all the uncertainties of the flavor problem. With this caution, we nevertheless conclude that the following rare decays are particularly worthy of further experimental searches:

$\mathrm{K}_{\mathrm{L}}^{0} \rightarrow \mu \mathrm{e}$,

This work is supported by the Department of Energy.

$$
\begin{aligned}
& \Sigma^{+} \rightarrow \mathrm{p} \mu \mathrm{e}, \\
& \tau \rightarrow \mu \mu \mathrm{e}, \\
& \mu \rightarrow \text { eee } .
\end{aligned}
$$

We note that (1) and (2) involve simultaneous flavor changes in both the quark and lepton sectors. $\Sigma^{+}$decay may have important advantages since it does not require coupling to a pseudoscalar current and is not sensitive to helicity suppression. Decay (3) requires flavor changes at both vertices of a boson exchange diagram while for (4) this occurs at only one vertex. Obviously, the above decays are not the only interesting ways of searching for rare flavor-changing processes. One can immediately think of others: neutrino oscillations, $\mu \rightarrow \mathrm{e} \gamma, \mu \rightarrow \mathrm{e}$ conversions on nuclei, $\nu$ reactions, $\mathrm{D} \overline{\mathrm{D}}$ or $\mathrm{B} \overline{\mathrm{B}}$ mixing. Indeed we would hope that all experimental avenues to the flavor problem will be explored with the best feasible sensitivity.

In section 2 we summarize the implications of the present experimental limits in the kaon system for searches of other decay processes. This requires a number of assumptions which, if changed, would clearly affect the conclusions. In section 3 we discuss the relevant experimental situation with emphasis on an estimate of achievable sensitivities. We conclude the paper in section 4 with a table that summarizes the various theoretical limits as well as present and future experimental sensitivities. Also listed for com- 
pleteness are other processes related to the flavor problem which are not discussed in detail in the text.

We note that Cahn and Harari [2] have also made an analysis of flavor-changing processes while this work was in progress.

2. Theoretical limits from existing information. In this section and for most of this paper we concentrate on the neutral couplings (s, d), $(\mu, \mathrm{e}),(\tau, \mu),(\tau, \mathrm{e})$ where sensitive experiments have been or can be carried out. As usual, the best restriction on the strength of such couplings comes from the $\mathrm{K}_{\mathrm{L}}-\mathrm{K}_{\mathrm{S}}$ mass difference. Assume, as in fig. $1 \mathrm{a}$, that boson $\mathrm{V}$ couples directly to s $\overline{\mathrm{d}}$ and $\overline{\mathrm{sd}}$. Then we obtain $\neq 1$

$\Delta m_{\mathrm{K}_{\mathrm{L}}-\mathrm{K}_{\mathrm{S}}} \approx\left(g_{\mathrm{sd}}^{2} / m_{\mathrm{V}}^{2}\right) f_{\mathrm{K}}^{2} m_{\mathrm{K}}$,

where $g_{\text {sd }}$ is the $V s \bar{d}$ coupling and we assume that $\left\langle 0\left|J_{\mathrm{V}}\right| \mathrm{K}\right\rangle$ is proportional to the usual $f_{\mathrm{K}}$. With $\Delta m /$ $m_{\mathrm{K}}=0.7 \times 10^{-14}$ and $f_{\mathrm{K}}^{2}=0.02 \mathrm{GeV}^{2}$ :

$g_{\text {sd }}^{2} / m_{\mathrm{V}}^{2} \lesssim \frac{1}{3} \times 10^{-12} \mathrm{GeV}^{-2}$,

or qualitatively $\neq 2$

$g_{\mathrm{sd}}^{2} / m_{\mathrm{V}}^{2}<10^{-7} G_{\mathrm{F}}$.

The direct $\mathrm{K}^{0}-\overline{\mathrm{K}}^{0}$ transition requires the boson $\mathrm{V}$ to

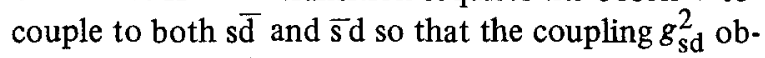
tained above might include a possible suppression factor associated with this mixing. Limits from $D \bar{D}$ mixing [5] give a comparable sensitivity for $g_{\mathrm{cu}}^{2} / m_{\mathrm{V}}^{2}$.

(a) $K_{L}^{O} \rightarrow \mu e$. Now, if $\mathrm{V}$ also couples to $\mu \mathrm{e}$ with a coupling $g_{\mu e}$, then we expect the rate for $\mathrm{K}_{\mathrm{L}} \rightarrow \mu \mathrm{e}$ (see fig. 1b) to be given by

$\Gamma\left(\mathrm{K}_{\mathrm{L}} \rightarrow \mu \mathrm{e}\right) \approx \frac{g_{\mathrm{sd}}^{2}}{m_{\mathrm{V}}^{2}} \frac{g_{\mu \mathrm{e}}^{2}}{m_{\mathrm{V}}^{2}} m_{\mu}^{2} f_{\mathrm{K}}^{2} m_{\mathrm{K}}$,

where $m_{\mu}^{2}$ is due to the helicity suppression expected in leptonic decays of a pseudoscalar particle and $f_{\mathrm{K}}$ is assumed to measure the $\mathrm{K}$ to vacuum transition. $\mathrm{Ex}$ perimentally [6] $\mathrm{BR}\left(\mathrm{K}_{\mathrm{L}} \rightarrow \mu \mathrm{e}\right) \lesssim 2 \times 10^{-9}$ so that $g_{\mu \mathrm{e}}^{2} / m_{\mathrm{V}}^{2} \lesssim 5 \times 10^{-10} \mathrm{GeV}^{-2}=5 \times 10^{-5} G_{\mathrm{F}}$.

The limits expressed in (7) and (9) above, can now be used to estimate limits for the branching ratio (BR) of other particle decays. We emphasize again that such

\footnotetext{
\$1 This result is consistent with the analysis of Applequist et al. [3].

$\neq 2$ This result appeared recently in ref. [4].
}

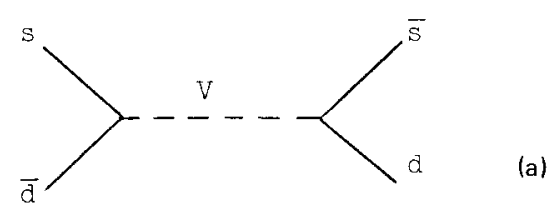

(a)

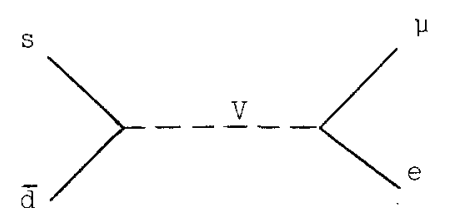

(b)
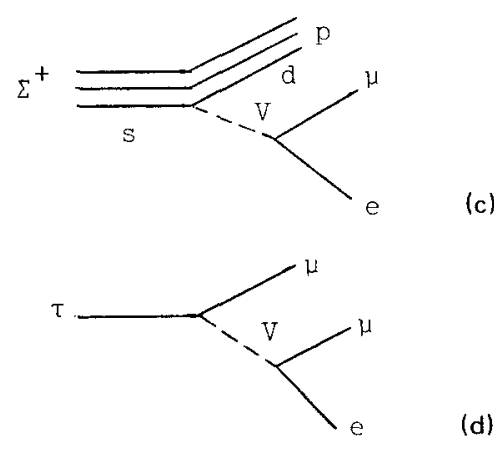

(d)

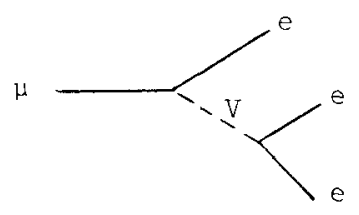

(e)

Fig. 1. Diagrams for particle decays described in text (fig. 1a is for $\mathrm{K}^{0}-\overline{\mathrm{K}}^{0}$ mixing).

estimates are necessarily uncertain since we do not know the details of neutral flavor-changing currents.

(b) $\Sigma^{+} \rightarrow p \mu e$. Using the diagram of fig. $1 \mathrm{c}$ and calculating with the simplifying assumption of massless leptons we find that the width for this decay goes as $\Delta^{2}=\left(m_{\Sigma}-m_{\mathrm{p}}\right)^{2}$ for $\mathrm{S}, \mathrm{P}$ and/or $\mathrm{V}, \mathrm{A}$ currents and is given approximately by

$\Gamma\left(\Sigma^{+} \rightarrow \mathrm{p} \mu \mathrm{e}\right) \approx \frac{g_{\mathrm{sd}}^{2}}{m_{\mathrm{V}}^{2}} \frac{g_{\mu \mathrm{e}}^{2}}{m_{\mathrm{V}}^{2}} \frac{m_{\Sigma}^{5}}{192 \pi^{3}}\left(\frac{\Delta}{m_{\Sigma}}\right)^{2}$,

yielding a branching ratio

$\operatorname{BR}\left(\Sigma^{+} \rightarrow \mathrm{p} \mu \mathrm{e}\right) \lesssim 10^{-12}$.

Note that, like the other limits quoted in this section, 
this is based on the $\mathrm{K}_{\mathrm{L}}-\mathrm{K}_{\mathrm{S}}$ mass difference and the present limit on $\mathrm{K}_{\mathrm{L}}^{0} \rightarrow \mu \mathrm{e}$. From a theoretical point of view this is a particularly attractive decay for an experimental search. It is not likely to be suppressed by an accident of the quantum numbers of the current. It mixes the same families and will occur in almost any model. For example, it is the only one of the decays we discuss that will occur in the scheme discussed by Veltman [1].

(c) $\tau \rightarrow \mu \mu e$ (also $\tau \rightarrow \mu \mu \mu$,eee, $\mu e e$ ). The width for this decay (fig. $1 \mathrm{~d}$ ) is given by

$\Gamma(\tau \rightarrow \mu \mu \mathrm{e}) \simeq \frac{g_{\tau \mu}^{2}}{m_{\mathrm{V}}^{2}} \frac{g_{\mu \mathrm{e}}^{2}}{m_{\mathrm{V}}^{2}} \frac{m_{\tau}^{5}}{192 \pi^{3}}$.

If we assume that $g_{\tau \mu}=g_{\mu \mathrm{e}}$ then we deduce a branching ratio limit of

$\mathrm{BR}(\tau \rightarrow \mu \mu \mathrm{e}) \approx 5 \times 10^{-10}$.

The $\tau$ decay modes may involve flavor change at only one vertex or at both. We assume them all to be of the same strength.

(d) $\mu \rightarrow e e e$. The diagram for this decay is given in fig. le. We assume here that the $\mathrm{V}$ bosons couple also to the non-flavor changing current $(e, e)$ with the same strength $\left(g_{\mathrm{ee}}=g_{\mu \mathrm{e}}\right)$. We obtain then:

$\Gamma(\mu \rightarrow$ eee $) \simeq\left(\frac{g_{\mu \mathrm{e}}^{2}}{m_{\mathrm{V}}^{2}}\right)^{2} \frac{m_{\mu}^{5}}{192 \pi^{3}}$

and

$\operatorname{BR}(\mu \rightarrow$ eee $) \lesssim 3 \times 10^{-9}$.

An important reason to search for $\mu \rightarrow$ eee would be to learn if the neutral currents in question contributed to both diagonal and off-diagonal flavor transitions. The decays of $\tau \rightarrow \mu \mu \mu$ and $\tau \rightarrow$ eee would, of course, serve the same purpose.

We conclude this section with the following comments. It is possible that the estimates for $\Sigma^{+}$and $\tau$ decay, (10) and (12), are more reliable than for $\mathrm{K}_{\mathrm{L}}$ because the three body decays assume a Vff vertex which will be present if anything we are discussing is relevant. The $\mathrm{K}_{\mathrm{L}} \rightarrow \mu \mathrm{e}$ transition, however, requires the direct coupling of a pseudoscalar to a current, which could be suppressed. For example $f_{\mathrm{K}}$ might be suppressed by a factor $m_{\mathrm{d}} / m_{\mathrm{s}}$ in the coupling to $\mathrm{V}$. A similar suppression $m_{\mathrm{e}} / m_{\mu}$ could occur at the $\mu \mathrm{e}$ vertex. The relevant current may not even couple to pseudoscalars. Within the kaon family searches for
$\mathrm{K}^{ \pm} \rightarrow \pi^{ \pm} \mu \mathrm{e}$ may thus provide information different from that obtained in $\mathrm{K}_{\mathrm{L}}^{0} \rightarrow \mu \mathrm{e}$. Information on $\mathrm{K}^{ \pm}$ decay is listed in table 1 of section 4 .

3. Experimental considerations. One can think of a large variety of experiments to study the flavor problem. Here we limit ourselves to a discussion of those experiments which constitute searches for the rare decay modes treated in section 2 . We make rough but realistic estimates of sensitivities to such decay modes. One purpose of this exercise was to see if any particular experiment would be more promising than the others. It turns out that, given the theoretical uncertainties, all of the experiments are worth pursuing. We emphasize that our estimates are essentially of the "back of the envelope" type and are thus not at the level of a serious proposal.

(a) $K_{L}^{0} \rightarrow \mu e$. We discuss this decay first because of the general importance of kaon decay to many physics questions and also because sensitive limits already exist: $\mathrm{BR}\left(\mathrm{K}_{\mathrm{L}}^{0} \rightarrow \mu \mathrm{e}\right)<2 \times 10^{-9}$. A more sensitive search ${ }^{\ddagger 3}$ requires two important ingredients, an intense neutral beam and excellent spectrometer resolution. Previous experiments have worked with $\mathrm{K}_{\mathrm{L}}^{0}$ beam intensities of around $10^{6}$ per pulse. It is conceivable that beams of $10^{7} \mathrm{~K}_{\mathrm{L}}^{0}$ could be produced at either Fermilab or Brookhaven if a reasonable fraction of the primary accelerator beam were dedicated to the experiment. Taking into account spectrometer acceptance and the typical running at a large laboratory one can then estimate that something of the order of $10^{11}$ $\mathrm{K}_{\mathrm{L}}^{0}$ could be observed yielding a $\mathrm{K}_{\mathrm{L}}^{0} \rightarrow \mu$ e sensitivity of $10^{-11}$ in the branching ratio. To achieve this, however, requires the elimination of $\mathrm{K}_{\mathrm{L}}^{0} \rightarrow \mu$ e candidates obtained from $\mathrm{K}_{\mathrm{L}}^{0} \rightarrow \pi \mathrm{e} \nu$ where the $\pi$ either decays to a muon or is misidentified as one and where the neutrino carries off very little c.m. energy. The " $\mu \mathrm{e}$ " mass endpoint from $\mathrm{K}_{\mathrm{L}}^{0} \rightarrow \pi \mathrm{e} \nu$ lies approximately $20 \mathrm{MeV}$ below the kaon mass and nearly all spurious events can be cut away if the mass resolution is optimized. To achieve the $10^{-11}$ branching ratio sensitivity will be very difficult but probably not impossible.

(b) $\Sigma^{+} \rightarrow p \mu e$. This decay probes essentially the same physics as $\mathrm{K}_{\mathrm{L}}^{0} \rightarrow \mu \mathrm{e}$ (but see remarks at end of section 2). No limits have been published. The search

${ }^{\ddagger 3} \mathrm{H}$. Frisch provided helpful information on $\mathrm{K} \rightarrow \mu \mathrm{e}$ experiments. 
for this decay mode is worthy of consideration since the physical background may be less than in $\mathrm{K}_{\mathrm{L}}^{0} \rightarrow \mu \mathrm{e}$ decay. The only obvious background is $\Sigma^{+} \rightarrow(\Lambda \rightarrow$ $\mathrm{p} \pi) \mathrm{e} \nu$ where the $\pi$ either decays to a muon or is misidentified as one and where the $\nu$ carries off little c.m. energy. We estimate this background to be less than $10^{-13}$ of all $\Sigma^{+}$decays. The background is small primarily because the branching ratio of $\Sigma^{+} \rightarrow \Lambda \mathrm{e} v$ is small $\left(\approx 10^{-5}\right)$ and because a $\Lambda$ in the final state is easily identifiable. So the availability of high intensity $\Sigma^{+}$beams becomes crucial to a sensitive search. We estimate ${ }^{\ddagger 4}$ roughly that at Fermilab energies between $10^{7}$ and $10^{8} \Sigma^{+}$per pulse might be observable in the decay region of a spectrometer if all beam parameters were optimized. This assumes $10^{12}$ protons per pulse on the primary production target. A branching ratio sensitivity of $10^{-12}$ to $10^{-13}$ for $\Sigma^{+} \rightarrow$ p $\mu$ e might thus be achievable during a major experimental run. A serious experimental problem would be background interactions from the $\approx 10^{9}-10^{10}$ protons per pulse accompanying the $\Sigma^{+}$beam. These would generate high detector rates.

(c) $\tau \rightarrow \mu \mu e(\tau \rightarrow \mu \mu \mu, e e e, \mu e e)$. The search for these decay modes of the $\tau$ lepton, best done at $\mathrm{e}^{+} \mathrm{e}^{-}$ storage rings, is limited by event rates and not backgrounds. Ideally one would do the search at a high

₹4 This estimate is based on information received from $O$. Overseth and J. Lach. luminosity machine at an energy not too high above threshold. The Cornell CESR machine might provide the most copious source of $\tau$-leptons. Assuming a typical luminosity of $10^{31} \mathrm{~cm}^{-2} \mathrm{~s}^{-1}$, one can expect to observe $\approx 10^{5} \tau$ 's after one to two years of data accumulation. This sets the sensitivity of the search at the $10^{-5}$ level. This compares unfavorably to the theoretical limit of $5 \times 10^{-10}$ deduced in section 2 . However, so little is known regarding rare decayse of heavy particles that an experimental search, even at the $10^{-5}$ level, is still desirable.

Since the $\tau$ is fully reconstructed in these decay modes one might also consider searching for them in $\tau$ 's produced in hadron collisions ${ }^{\ddagger 5}$ such as at Fermilab. One possible source of $\tau$ 's is $F$ decay. If we make the crude assumptions that $\sigma_{\mathrm{F}} \approx 1 \mu \mathrm{b}$ and $\mathrm{BR}(\mathrm{F} \rightarrow$ $\tau \nu$ ) $\approx 1 \%$ then we expect $\approx 10^{-7} \tau$ 's per interacting proton. To reconstruct $\tau \rightarrow \mu \mu$ e would require an "open" spectrometer configuration (i.e. no hadron absorber) in order to detect the electrons and to achieve good mass resolution. This necessarily limits beam rates to something like $10^{6}-10^{7}$ interacting protons per pulse. A major experiment might thus be sensitive to $\approx 10^{5}$ decays - a number similar to that achievable in the $\mathrm{e}^{+} \mathrm{e}^{-}$storage rings. A serious source of background events originates from hadronic di-

₹5 This was suggested by J.D. Bjorken.

Table 1

Summary of limits and sensitivities.

\begin{tabular}{|c|c|c|c|c|c|}
\hline Process & Present limits [9] & $\begin{array}{l}\text { Limit suggested } \\
\text { by } \mathrm{K}^{0} \text { data }\end{array}$ & $\begin{array}{l}\text { Achievable } \\
\text { exp. sensitivity }\end{array}$ & $\begin{array}{l}\text { Experiment } \\
\text { limited by }\end{array}$ & Comments \\
\hline$\Delta m\left(\mathrm{~K}_{\mathrm{L}}-\mathrm{K}_{\mathrm{S}}\right)$ & $g_{\mathrm{Sd}}^{2} d m<10^{-7} G_{\mathrm{F}}$ & & & & strongest present limit \\
\hline $\mathrm{K}_{\mathrm{L}} \rightarrow \mu \mathrm{e}$ & $\left\{\begin{array}{l}\mathrm{BR}<2 \times 10^{-9} \\
g_{\mu \mathrm{e}}^{2} / m^{2}<10^{-5} G_{\mathrm{F}}\end{array}\right.$ & $\mathrm{BR}<2 \times 10^{-9}$ & $\mathrm{BR} \approx 10^{-11}$ & $\mathrm{~K} \rightarrow \pi \mathrm{e} \nu, \mathrm{bkg}$ & $\begin{array}{l}\text { requires pseudo- } \\
\text { scalar coupling }\end{array}$ \\
\hline $\mathrm{K}^{+} \rightarrow \pi^{+} \mu \mathrm{e}$ & $\mathrm{BR}<5 \times 10^{-9}$ & $\mathrm{BR}<10^{-11}$ & $\mathrm{BR} \approx 10^{-10}$ & $\mathrm{~K} \rightarrow \pi \pi \mathrm{e} \nu$, beam & $\begin{array}{l}\text { limit estimated as } \\
\text { for } \Sigma \rightarrow \text { p } \mu \text { e }\end{array}$ \\
\hline$\Sigma^{+} \rightarrow$ p $\mu \mathrm{e}$ & none & $\mathrm{BR}<10^{-12}$ & $\mathrm{BR} \approx 10^{-12}-10^{-13}$ & beam, singles rates & $\begin{array}{l}\text { less model-dep. than } \\
\mathrm{K}_{\mathrm{L}} \rightarrow \mu \mathrm{e}\end{array}$ \\
\hline$\tau \rightarrow \mu \mu \mathrm{e}$ & none & $\mathrm{BR}<5 \times 10^{-10}$ & $\mathrm{BR} \approx 10^{-5}$ & luminosity & also $\mu \mu \mu$, eee, $\mu$ ee \\
\hline$\mu \rightarrow$ eee & $\mathrm{BR}<2 \times 10^{-9}$ & $\mathrm{BR}<3 \times 10^{-9}$ & $\mathrm{BR} \approx 10^{-12}-10^{-13}$ & beam, trigger & tests diagonal coupling \\
\hline $\mathrm{D} \rightarrow \pi \mu \mathrm{e}$ & none & similar to $\tau$ & $\mathrm{BR} \approx 10^{-5}$ & luminosity & \\
\hline $\mathrm{F} \rightarrow \mathrm{K} \mu \mathrm{e}$ & none & similar to $\tau$ & $\mathrm{BR} \approx 10^{-4}$ & luminosity & \\
\hline$\mu \rightarrow \operatorname{e\gamma }[10]$ & $\mathrm{BR}<2 \times 10^{-10}$ & & $\mathrm{BR} \approx 10^{-12}$ & $\mu \rightarrow$ e $\gamma \nu \nu$, beam & fmay be higher order \\
\hline$\mu \mathrm{N} \rightarrow \mathrm{eN}[11]$ & $\begin{array}{l}4 \times 10^{-10} \text { rela- } \\
\text { tive to } \mu \text { capture }\end{array}$ & & $\approx 10^{-12}$ & $\mu \rightarrow \mathrm{e}_{\nu v}$, beam & than $\mu \rightarrow$ eee \\
\hline$\Theta^{0} \rightarrow \Lambda^{0} \mu \mathrm{e}$ & none & similar to $\Sigma^{+}$ & $\mathrm{BR} \approx 10^{-11}$ & beam & $\begin{array}{l}\text { physics similar to } \\
\Sigma^{+} \rightarrow \text { p } \mu \mathrm{e}\end{array}$ \\
\hline
\end{tabular}


muon production accompanied by electrons from $\pi^{0}$ Dalitz decay. We estimate that the branching ratio sensitivity for $\tau \rightarrow \mu \mu$ e produced in hadron collisions is at the $10^{-4}$ level.

(d) $\mu \rightarrow$ eee. Presently a major experimental effort [7] is underway at LAMPF to search for this decay mode with a sensitivity at the $10^{-11}$ level in the branching ratio. The sensitivity will probably be limited by beam intensity, spectrometer solid angle and triggering capacity, and not by the physical backgrounds. Conceivably, this kind of experiment could be pushed one or more orders of magnitude to the $10^{-12}$ to $10^{-13}$ level ${ }^{\ddagger 6}$.

4. Conclusions and comments. In the previous sections we have focussed on four rare decay processes for the search of neutral flavor-changing processes. It is very interesting to note that in the best conceivable experiments the limits on $\left(g^{2} / m_{\mathrm{V}}^{2}\right)^{2}$ can be improved by several orders of magnitude. This information is summarized in table 1 . We believe that it is worthwhile to pursue all of these rare decays in future experiments. We note that the achievable experimental sensitivities often fall within a range where some theore tical ideas suggest effects might be found [1]. From a theoretical point of view $\Sigma^{+} \rightarrow$ p $\mu$ e may be a particularly attractive process to study.

In table 1 we also list other processes of relevance to the flavor problem. Decays, such as $\mathrm{D} \rightarrow \mu \pi \mathrm{e}, \mathrm{F} \rightarrow$ $\mathrm{K} \mu \mathrm{e}$, or $\mu \rightarrow \mathrm{e} \gamma$, clearly address the same kinds of physics as those mentioned above. In addition, precise measurements of the $Z^{0}$ width may tell us the number of neutrino flavors. The discovery of other flavors beyond the $\tau$ lepton and the b quark would obviously

\#6 It might be possible to achieve $10^{-13}$ at Los Alamos [8]. add new information to the problem. It would certainly be nice to have more experimental input to help find why and how nature chooses to replicate its fermion families.

We have enjoyed various discussions on these topics with C. Akerlof, J. Bjorken, H. Frisch, O. Overseth, M. Veltman, T. Sterling, and others. We thank them for their interest. We thank R. Cahn for pointing out an error in an earlier version.

\section{References}

[1] For recent discussions of the flavor problem, or models with estimates of rates, see:

M. Gorn, Phys. Rev. D20 (1979) 2380;

A. Davidson, M. Koca and K.C. Wali, Phys. Rev. D20

(1979) 1195 ;

E. Derman, Phys. Rev. D19 (1979) 317;

M. Veltman, Invited Talk at Lepton and photon Conf. (Batavia, Aug. 1979);

E. Eichten and K. Lane, Dynamical breaking of weak interaction symmetries, HUTP-79/A062, to be published.

S. Dimopoulos, Technicolor signatures, to be published.

[2] R. Cahn and H. Harari, to be published.

[3] T. Applequist, J.D. Bjorken and M. Chanowitz, Phys. Rev. D7 (1973) 2225.

[4] E. Eichten and K. Lane, Dynamical breaking of weak interaction symmetries, HUTP-79/A062, to be published; V. Barger and E. Ma, preprint UW-C))-881-114, UH511-366-79, to be published.

[5] M. Gorn, Phys. Rev. D20 (1979) 2380;

E. Paschos, Phys. Rev. Lett. 39 (1977) 858.

[6] A.R. Clark et al., Phys. Rev. Lett. 26 (1971) 1667.

[7] Chicago-LASL-Stanford Collaboration.

[8] M. Duong-Van, provite communication.

[9] Based on data in Review of particle properties (April 1978).

[10] J.D. Bowman et al., Phys. Rev. Lett. 42 (1979) 556.

[11] A. Badertscher et al., Phys. Rev. Lett. 39 (1977) 1385. 